\title{
Un territorio y un río metropolitanos: \\ Buenos aires y el Paraná.
}

\section{A metropolitan territory and a metropolitan river: \\ Buenos Aires and the Paraná}

\author{
Thomas Massin * \\ Instituto Superior de Urbanismo. Universidad de Buenos Aires, Argentina / \\ Fundación Furban \\ tommassin@gmail.com
}




\title{
Resumen
}

Este artículo estudia la relación entre lo fluvial y lo urbano desde un enfoque regional, abarcando la metrópolis de Buenos Aires y el río Paraná, en su tramo inferior. Éste constituye un objeto geográfico complejo, a la vez río, litoral, delta y "autopista marítima" para los barcos de ultramar. Esta singularidad explicaría la dificultad de representación y de planificación de la metrópolis de Buenos Aires en articulación con su gran entorno natural. Por lo tanto, suponemos que se trata de un "espacio no concebido", retomando la teoría lefebvriana. Para tratar de superar esta aporía, el artículo introduce la figura de "metrópolis fluvial"e insiste en la doble dimensión medioambiental y operativa, unida por la dimensión de representación, para integrar el Paraná en las reflexiones metropolitanas creando un sentimiento de pertenencia colectiva y una identidad territorial.

Palabras clave: Área Metropolitana de Buenos Aires; río Paraná; representación; planificación urbana

\begin{abstract}
In thisarticle, westudytherelationshipbetween fluvial and urbandimensionsfrom a regional approach, coveringthemetropolis of Buenos Aires and the Paraná River, in itslowersection. Thelatteris a complexgeographicalobject, at thesame time a river, a coast, a delta, a "maritimehighway" foroverseasships. Thisoriginalitycouldexplainthedifficulty of representing and planningthe Buenos Aires metropolis in articulationwithitsgreat natural environment. Therefore, weassumethatitis a "notconceivedspace", taking up theLefebvriantheory. To try toovercomethisaporia, thearticleintroduces the figure of "fluvial metropolis" and insistsonthedoubleenvironmental and operationaldimension, unitedbythedimension of representation. Thisshouldhelptointegratethe Paraná intothereflectionson metropolitan processes, creating a feeling of collectivebelonging and a territorial identity.
\end{abstract}

Keywords: Buenos Aires'Metropolitan Area; Paraná River; representation, urban planning 


\section{Introducción}

La visita en mayo de 2017 a Buenos Aires del urbanista francés reconocido por sus trabajos sobre el Gran París y el Gran Moscú, AntoineGrumbach, volvió a poner de manifiesto la importancia de la "geografía", como medio natural, en la construcción tanto simbólica y política como operativa de las áreas metropolitanas. En el caso del Área Metropolitana de Buenos Aires (AMBA) y del corredor metropolitano que la conecta a Rosario, este enfoque abre a una reflexión rica. En efecto, la proximidad del río Paraná, soporte de actividades industriales pesadas, y de su Delta, que alberga una población muy vulnerable, a estas dos metrópolis, lo convierte en una pieza clave para la planificación metropolitana. Tener en cuenta el Paraná como elemento territorial estructurador permite salir de la visión meramente funcional o mitificada, heredada de la figura del eje fluvial industrial, que impide la comprensión de las lógicas espaciales y de los desafíos sociales y medioambientales que allí se ponen en juego. Por lo tanto, en este artículo estudiamos la relación entre lo fluvial y lo urbano desde un enfoque regional, abarcando la metrópolis de Buenos Aires, el río Paraná en su curso inferior y su Delta. Más específicamente, queremos demostrar que las representaciones cartográficas e iconográficas que existen de este territorio hacen que sea difícil considerarlo para la planificación. Proponemos entonces la figura de metrópolis fluvial para tratar de superar estas dificultades.

\section{La representación del río y del delta a escala metropolitana: las dificultades conceptuales}

El Paraná y su Delta y la metrópolis, los parientes pobres de la planificación

Si bien la mancha metropolitana se representa por primera vez en un documento de planificación en 1925, el Plan Regulador y de Reforma de la Ciudad elaborado por la Comisión de Estética Edilicia, es en la década de los 1960 cuando aparecen los primeros planes y planos para la región metropolitana. Así en 1962 se publica el Plan Regulador de la Ciudad de Buenos Aires (Municipalidad de Buenos Aires, 1962), que
Figura 1: Plan Regulador de la Ciudad de Buenos Aires

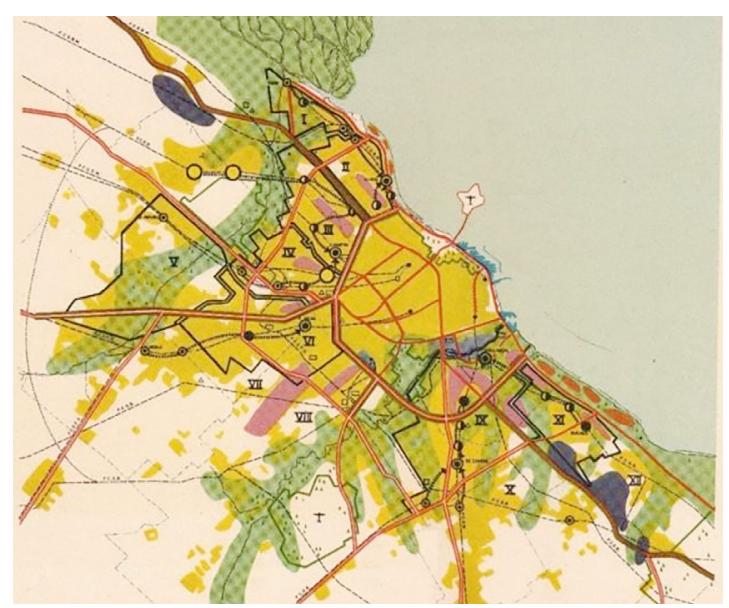

Fuente: Municipalidad de Buenos Aires (1962)

adopta tres escalas de planeamiento. Una corresponde al Área Urbana, equivalente a la jurisdicción de la CABA; otra al Área Metropolitana, conformada por la CABA y los sectores urbanos de los partidos limítrofes en un radio de $30 \mathrm{~km}$; la última al Área Regional que comprende el Área Metropolitana y los partidos en un radio de 100 km (fig. 1).

En paralelo, aparece el concepto de eje fluvial industrial. Después de varias referencias a la industrialización del litoral en el Primer Plan Quinquenal (1946-1951), se usa el término en el Esquema Director Año 2000 (Presidencia de la Nación, 1969), un documento de planeamiento a escala regional que orienta la visión del área por casi dos décadas. En este esquema se hace hincapié en la organización espacial de la metrópolis, basándose en la hipótesis que la misma continuaría creciendo de manera lineal, y se consolida la visión regional sobre el borde fluvial, proyectándose "ejes preferenciales de urbanización”. Más precisamente, se considera al territorio comprendido por Capital Federal y 25 partidos de la Provincia de Buenos Aires como una faja costera donde se desarrolla una metrópolis lineal entre dos ejes paralelos de urbanización que dibujan una franja de $20 \mathrm{~km}$ de ancho entre La Plata, Buenos Aires y Rosario. Esta expansión metropolitana lineal respeta la configuración natural de la costa del Paraná y del Río de la Plata y se opone a la configuración radio concéntrica y centralizada tradicional (fig. 2). 
Sin embargo, en estos dos documentos, el papel dado al Paraná y al Delta en su relación con la metrópolis resulta poco preciso. Una expresión clara de eso es la manera de tener en cuenta la superficie insular -o deltaica- de los partidos que integran la metrópolis. ${ }^{1}$ Por ejemplo, en el censo de población de 1970 (INDEC, 1970), el mapa del Gran Buenos Aires integra la parte insular de Tigre y de San Fernando. Es el caso también de los documentos de planificación de la Provincia de Buenos Aires, como el del Ministerio de Infraestructura, Vivienda y Servicios Públicos (2006). Si desde un punto administrativo esta delimitación parece lógica, desde un punto de vista geográfico y funcional conlleva serios problemas. Otros documentos de planificación no aportan mucha clarificación a este situación, ya sean el Plan Urbano Ambiental (Consejo del Plan Urbano Ambiental, 1999) o el Estudio para la Formulación del Esquema Director Intermunicipal del Borde Metropolitano (Provincia de Buenos Aires, 2010). Finalmente, en cambio, desde la Subsecretaría de Urbanismo y Vivienda de la Provincia de Buenos Aires (2007) existe una voluntad, aunque meramente formal, de separar los territorios insulares de los partidos, integrando el Delta. Esta situación ambigua simboliza la

1. Tigre, San Fernando, Escobar, Zárate, Campana (donde la parte deltaica representa los dos tercios de la superficie del partido).
Figura 2: El eje fluvial industrial, Esquema director Año 2000

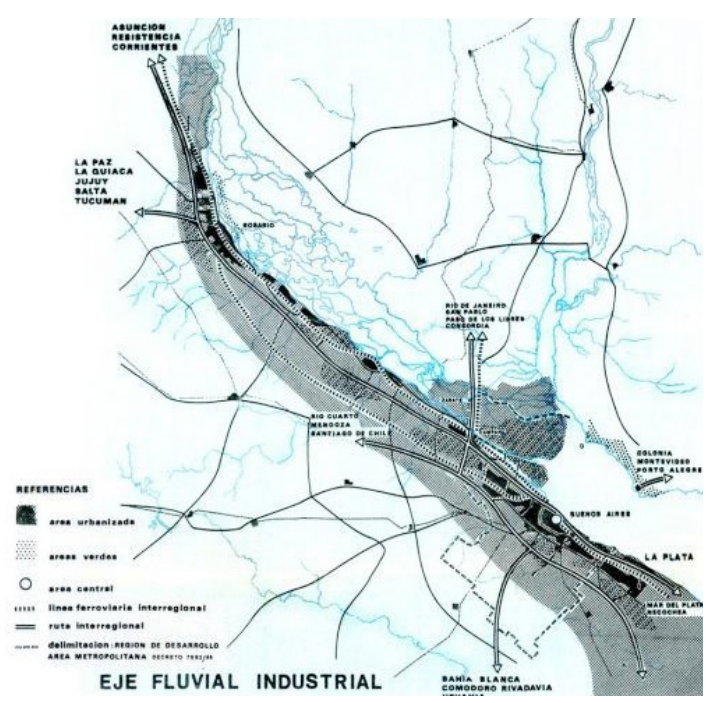

Fuente: Presidencia de la Nación (1969)

dificultad de representación y por lo tanto de planificación de la metrópolis en articulación con uno de sus grandes entornos naturales (con la llanura pampeana).

El documento final del Plan Urbano Ambiental contempla los espacios abiertos de escala regional. Más precisamente, propone elaborar un plan de manejo del Delta del Paraná, con participación plural que incluya a la población isleña, que conjugue la preservación de sus carac-

Figura 3: Plan Urbano Ambiental - Documento final

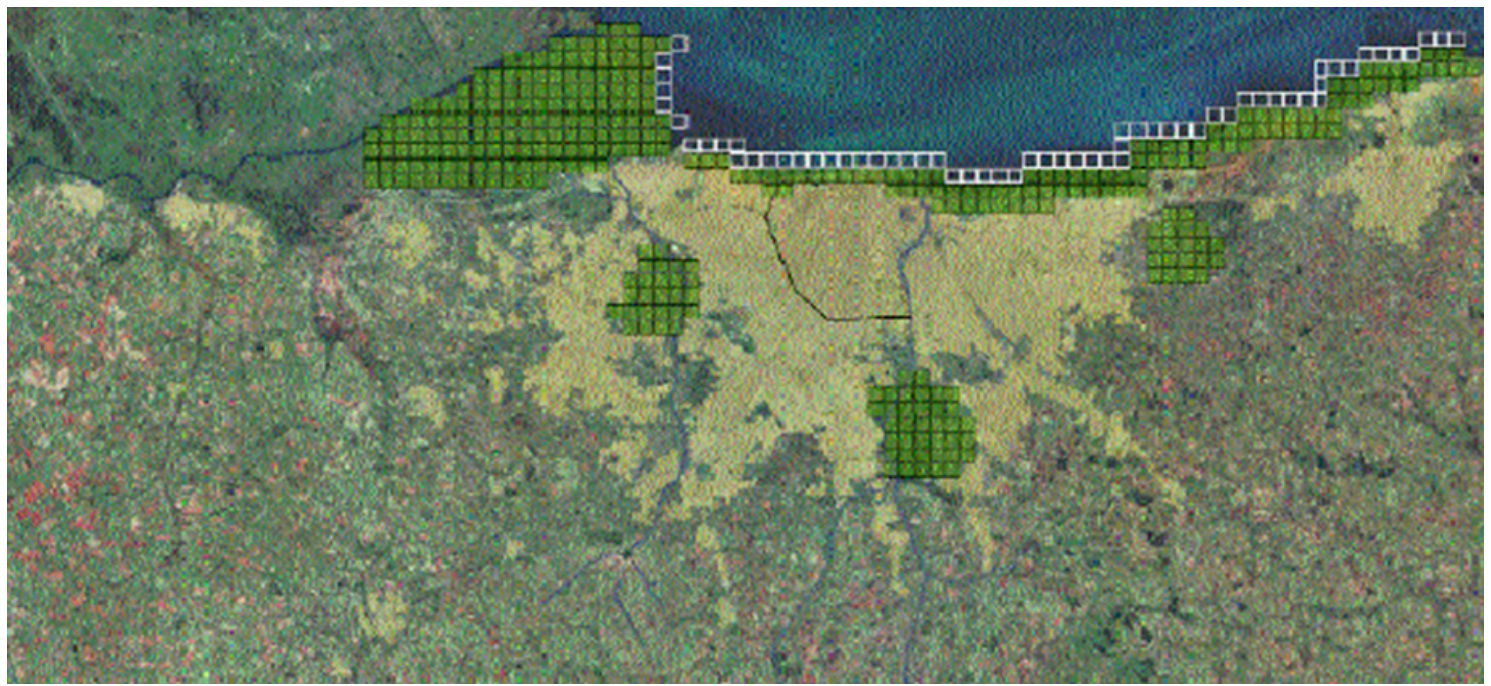

Fuente: Consejo del Plan Urbano Ambiental (2000) 
Figuras 4 a y b: Lineamientos Estratégicos para la Región Metropolitana de Buenos Aires
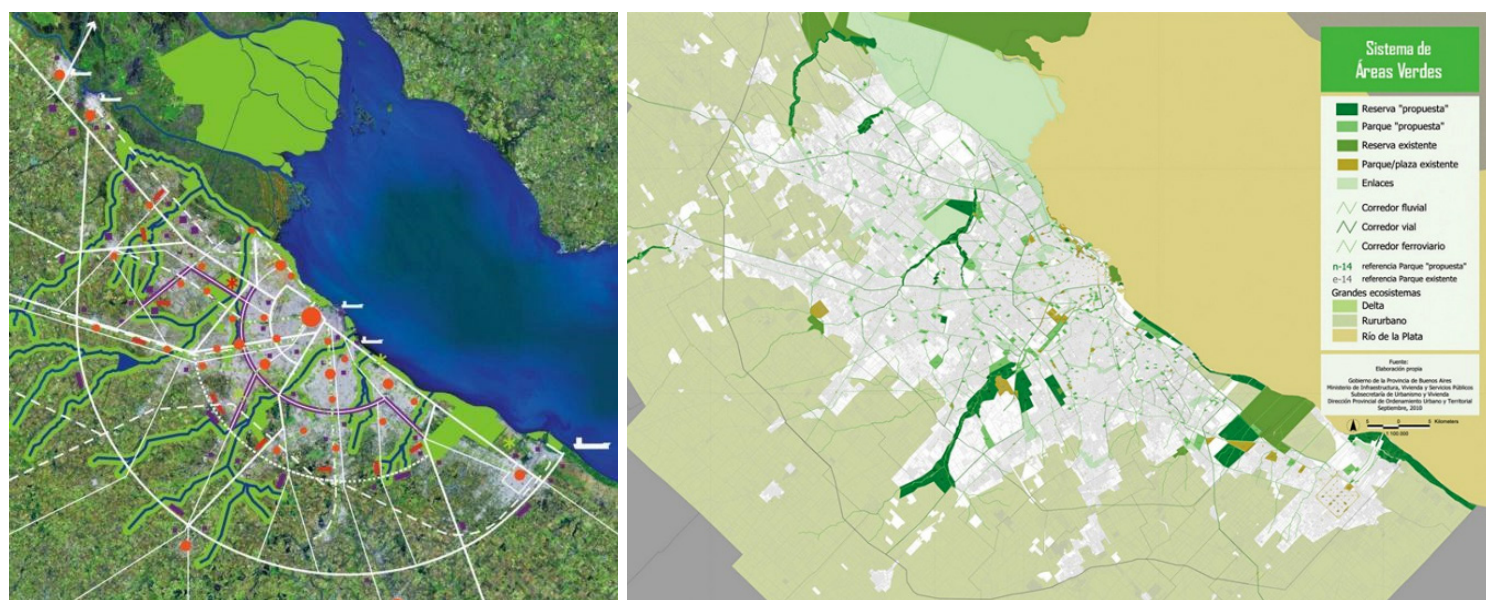

Fuente: Subsecretaría de Urbanismo y Vivienda de la Provincia de Buenos Aires (2007)

terísticas naturales singulares con un desarrollo socio productivo controlado, y que tenga en cuenta los convenios internacionales de defensa de los humedales. Sin embargo, a pesar de estas intenciones, el documento no distingue gráficamente el Delta de la costanera o de los otros espacios abiertos de gran escala (fig. 3).

A su vez, los Lineamientos Estratégicos para la Región Metropolitana de Buenos Aires sostienen que la potencia natural y el grado de conservación del Delta del Paraná en contacto con la zona urbana del Gran Buenos Aires es un privilegio que pocas ciudades poseen. Sin embargo, la amenaza de impactos irreversibles por un uso indebido es obvia. "Preservar esta gran reserva verde es primordial y las figuras de protección propuestas para el conjunto del mismo deberían contar con planificación específica para regular los usos y preservar el capital biológico del Delta" (Subsecretaría de Urbanismo y Vivienda de la Provincia de Buenos Aires, 2007:189). Se propone, por lo tanto, orientar estas áreas al desarrollo de usos turístico recreativos, productivos, científicos, educativos, etc. evaluando los impactos positivos y negativos de la propuesta. Desde un punto de vista gráfico, sin embargo, las propuestas específicas para el Delta no se representan de manera clara: la reserva de biosfera está coloreada con un verde más claro que el resto del Delta, que no está categorizado (fig. 4a). En otro mapa del documento, esta parte se considera como "enlace" y "espacio de recreación" (fig. 4b).

Finalmente, el Estudio para la Formulación del Esquema Director Intermunicipal del Borde Metropolitano (Provincia de Buenos Aires, 2010) tampoco ofrece una consideración clara del Delta, cuando nos parece que es un elemento central de la planificación de las periferias metropolitanas.

Por lo tanto, a lo largo de los últimos 60 años, en los documentos de planificación, publicados desde el Estado federal, la Provincia de Buenos Aires o la CABA, se destaca la dificultad (asumida o no) de integrar el Delta y el Paraná en la planificación metropolitana. Y cuando se hace, es de una manera confusa y en general funcionalista. Identificamos tres tipos de dificultades que pueden explicar esta situación: la terminología, el imaginario, la escala.

\section{Las razones de estas dificultades conceptuales}

El término río resulta insuficiente para connotar las singularidades geográficas del Paraná: en su tramo inferior lindante a la metrópolis de Buenos Aires, no es un mero curso de agua, ni solamente un Delta, inmenso espacio abierto a pocos kilómetros del foco metropolitano. El alemán y el francés, entre otros idiomas, distinguen 
Figura 5: Detalle del mapa MundusNovus de Diego Ribero en 1529

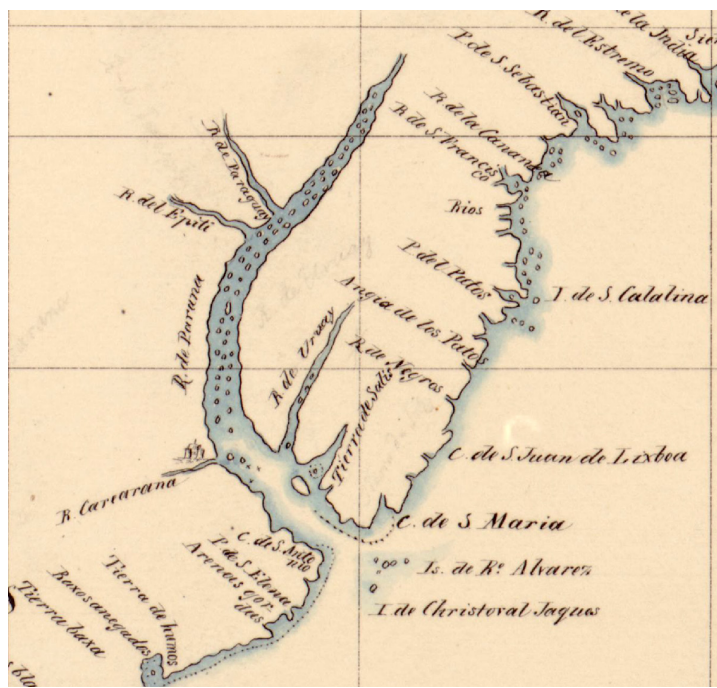

Fuente: copia en la Biblioteca del Congreso de Estados Unidos

claramente entre fluss / rivière y Strom / fleuve, entre los cursos de agua según su importancia y si desemboca o en otro río o en el mar. En español, como en inglés, se usa el término río de manera genérica. ${ }^{2}$ Estaausencia léxicaimpide que se considere con claridad el Paraná, más aún cuando se trata de un megariver (Latrubesse, 2008). En esta categoría se encuentran los ríos con caudal medio anual superior a los $17.000 \mathrm{~m} 3 / \mathrm{s}$ y caracterizados por la existencia de brazos entre islas aluviales. ${ }^{3}$ La misma confusión ocurre con el término litoral, que se usa con frecuencia para hablar de la margen derecha del Paraná, cuando en los restantes países de habla hispana se reserva exclusivamente para el borde marino (Dadon, 2003). Esta ambigüedad terminológica es geográfica e histórica, pero también refleja la naturaleza atípica del $\mathrm{Pa}$ raná cuyo calado lo convierte en una "autopista marítima" para los barcos de ultramar, a semejanza de un litoral marítimo.

Si bien las primeras representaciones cartográficas del Paraná datan del siglo XVI (Forget, 2011) (fig. 5), existen relativamente pocos datos

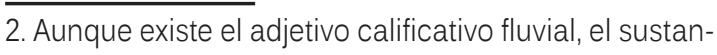
tivo asociado no se usa en castellano.

3. En Sudamérica, los ríos Paraná, Amazonas y Orinoco.
Figura 6: llustración de un libro de lectura para primer grado inferior

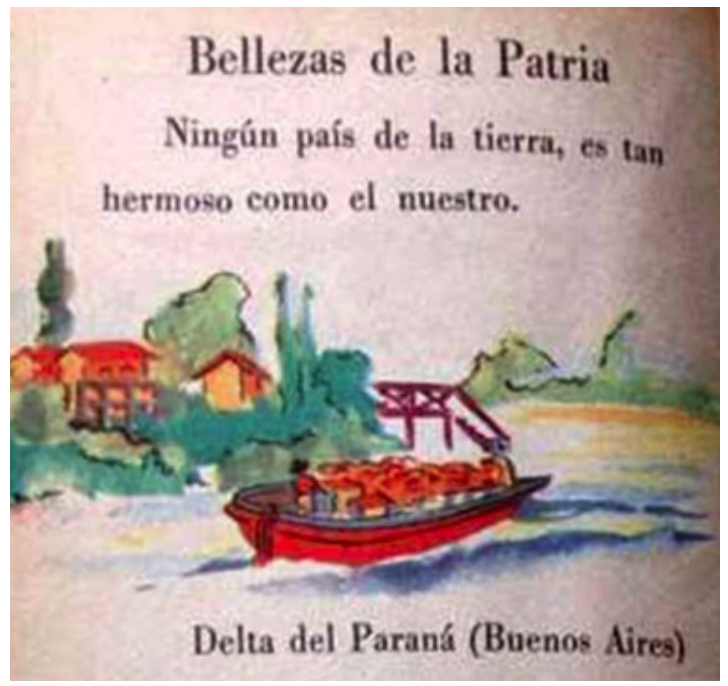

Fuente: Albornoz Videla (1950)

y trabajos de investigación en el campo de las ciencias humanas sobre el río y su Delta. Eso favorece la existencia de un imaginario colectivo, alimentado por relatos antiguos y míticos (fig. 6), resumido por Rodolfo Walsh (1995:198): "Una región casi tan extensa como la provincia de Tucumán espera ser conquistada por segunda vez. Cercano y desconocido, el Delta del Paraná revive la odisea de sus pioneros". Así, para las instancias públicas, el Delta es un espacio singular y el lema de los documentos de planificación ha evolucionado desde su "modernización" hasta su "desarrollo" y "fomento", entre mediados del siglo XX y los años 2000. En cambio, desde hace una década se promueve su "conservación" y "aprovechamiento".

A su vez, la escala considerable de la metrópolis de Buenos Aires y del megariver Paraná y de su Delta, dificultan la representación cartográfica de este territorio: presentan características que superan los límites de los objetos río y ciudad, en tres dimensiones (Monnet, 2001):

- superación de las capacidades perceptivas del individuo: la metrópolis y el Delta no pueden ser abarcados por la mirada del individuo, no pueden ser recorridos totalmente, no pueden ser conocidos de manera familiar en su 
conjunto. En efecto la escala que permite representar a la vez el AMBA y el Delta se encuentra entre $1 / 500.000$ y $1 / 1.000 .000$.

- superación de los límites de las unidades de gestión, de administración del territorio: no existe un cuerpo político-administrativo que pueda representarlos como conjunto y unidad;

- superación de las posibilidades de conceptualización unitaria, ya que los límites dados por criterios de la continuidad del tejido urbano y las unidades geomorfológicas no coinciden con la articulación funcional ni con el área de influencia.

En definitiva, es muy difícil pensar la metrópolis y su entorno natural, ya que están constituidos en gran parte por territorios escasamente conocidos y erróneamente conceptualizados. Dicho de manera directa, no se sabe muy bien qué hacer con el Delta y el Paraná y esta situación ambigua favorece a los actores y los intereses privados. Por lo tanto, nos parece muy importante tratar de superar estas limitaciones y considerar el Delta como interfaz con la metrópolis. Para eso, el concepto de "tercer espacio" es una buena herramienta heurística.

\section{Superar estas dificultades por el tercer espacio}

\section{Representar el tercer espacio}

La noción de tercer espacio (Vanier, 2000, 2003) permite considerar las periferias metropolitanas, el Delta en primer lugar, como un territorio específico. Es decir, que combina y supera las categorías clásicas para imaginar nuevas formas de gestión y de planificación. En efecto, los espacios periféricos metropolitanos tienen necesidades urbanas sin tener las características de densidad urbana. Además, presentan algunas especificidades de los espacios rurales, en las zonas agrícolas, forestales, húmedas y naturales que los componen. Esta situación implica que las periferias metropolitanas no entran en una lectura binaria del espacio. De ahí surge la necesidad de superar las dicotomías tradicionales: urbano / rural, centro / periferia, por ejemplo. Vanier le da una dimensión material, funcional y política al concepto; de esta forma el tercer espacio hace eco de la triplicidad del espacio, conceptualizada por Lefebvre (1974).

Lefebvre propone pensar el espacio en términos de representaciones, de usos, de prácticas y de apropiación. Esta teoría, desarrollada en la década de los 1970 en Francia, va en contra de la "cultura" de esa época: la del arquitecto, el urbanista y el tecnócrata, defensores de un espacio abstracto, determinado sin tener en cuenta las prácticas sociales. De manera sintética, mientras que el "espacio percibido" refleja la práctica espacial de una sociedad y el "espacio vivido" es aquel practicado por los individuos, el "espacio concebido" -el que aquí nos interesa- lo es por los políticos, los planificadores, los urbanistas, los tecnócratas, los expertos. Se trata de las representaciones del espacio. Para Lefebvre, es el espacio dominante en una sociedad, vector de ideologías.

Esta propuesta de Lefebvre tuvo mucha influencia y fue retomada y actualizada por numerosos autores: Edward Soja en primer lugar o el geógrafo francés Moine (2006) quien analiza el territorio identificando tres subsistemas en interacción. Además de la referencia espacial que es el espacio geográfico en su dimensión material y el sistema de los actores, la dimensión simbólica es central: son los sistemas de representación del espacio geográfico, constituidos por filtros individuales, sociales e ideológicos.

De estos aportes teóricos podemos concluir que:

- La representación del Delta y del Paraná es un desafío para la planificación de la metrópolis: el espacio concebido o la representación del espacio es fundamental.

- Hay que evitar de caer en una planificación funcional, centralizada, autoritaria, tecnócrata, que sirva al interés de los actores dominantes, en una lectura inspirada por Lefebvre.

- Por lo tanto, para pensar el Delta y el Paraná desde una visión metropolitana, hay que superar la visión tradicional y considerarlo como un territorio específico y central.

Además de varios estudios y propuestas que se han hecho en otras metrópolis del mundo que 


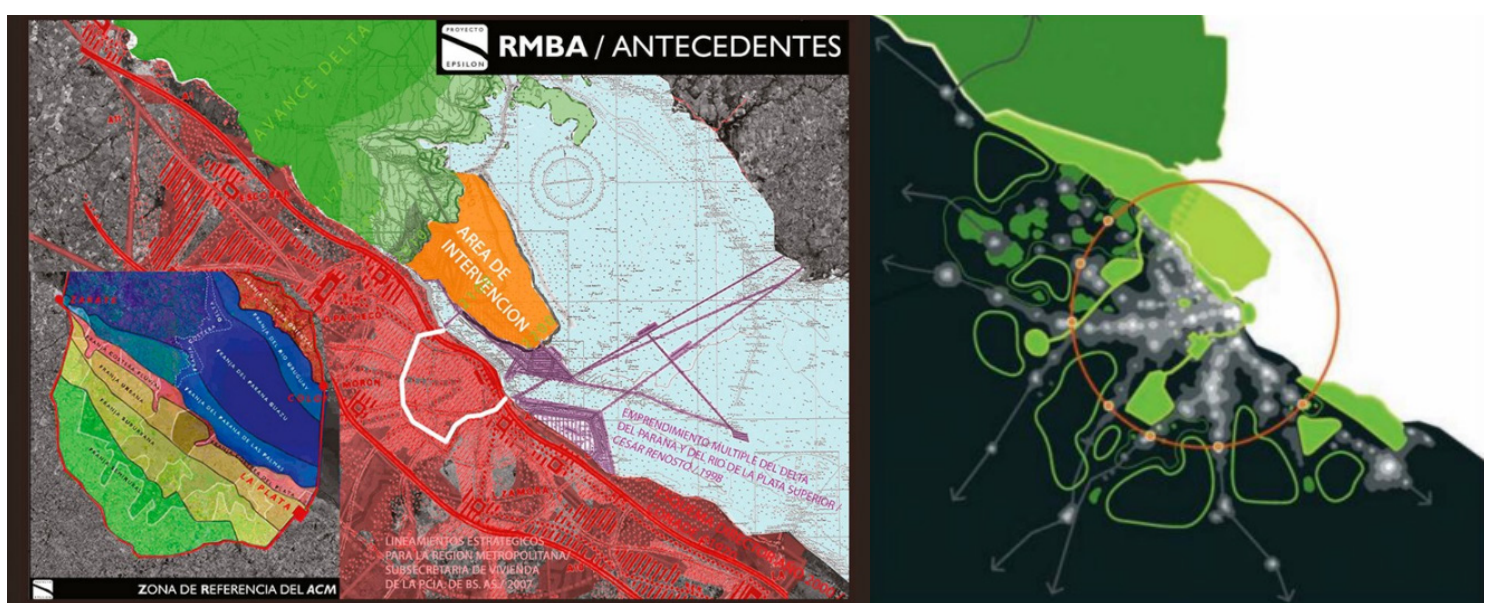

Fuente: Subsecretaría de Ambiente y Desarrollo Sustentable de la Nación (2002) y DellaPaolera (2010)

hacen hincapié en la representación espacial del río como elemento metropolitano central (París, Moscú, Montreal, entre otras), dos propuestas gráficas y proyectuales de arquitectos argentinos son pistas interesantes.

\section{Dos propuestas de representación}

La hipótesis de partida del Proyecto Epsilon de Claudio DellaPaolera (fig. 7) es que la sustentabilidad del desarrollo urbano del AMBA depende del manejo apropiado de la franja costera del Río de la Plata. En efecto, sostiene que la contaminación del Río de la Plata es una amenaza para la salud, que se ocupan 2.600 hectáreas de suelos agrícolas por año y que el Delta avanza 50 metros por año. Además, afirma que la ausencia de políticas integrales de manejo de la costa metropolitana evidencia que no se asumió la importancia de la condición de asentamiento costero, inhibiendo el desarrollo bajo condiciones de calidad ambiental. Propone entonces pensar el humedal del río como oportunidad y no como amenaza, incorporando un territorio fluvial de 12.000 hectáreas al planeamiento metropolitano, donde podrían localizarse parques, equipamientos deportivos, infraestructura ambiental y de transporte. ${ }^{4}$

4. Sin embargo esta propuesta de construir equipamientos o
A su vez el análisis de Martín Menini(fig. 8) destaca la situación del AMBA en un verdadero ecotono, zona fronteriza entre distintos ecosistemas naturales: la Pampa húmeda, el Delta del Paraná, y el Río de la Plata. Eso "significa un enorme potencial de desarrollo sustentable para consolidar su rol estratégico frente a crecimiento exponencial de Brasil como nueva potencia mundial" (Menini, 2013:52). El proyecto propone la preservación de los ecosistemas naturales: delta, humedales, dunas, ríos, bosques.

Estas dos pistas plantean ideas muy interesantes, que proponemos profundizar insistiendo en la doble dimensión medioambiental y operativa, unida por la dimensión de representación.

\section{La propuesta de la metrópolis fluvial}

El papel del Delta en la metrópolis: dimensión operativa, dimensión medioambiental y representación

Desde un punto de vista operativo, los grandes ríos navegables, el Paraná especialmente, son soportes fundamentales de los flujos globalizados y sus puertos son el lugar de interfaz entre lo local y lo global (Forget, 2011; Massin, 2017).

$\overline{\text { infraestructuras sobre las tierras deltaicas nos parece un tanto }}$ arriesgada. 


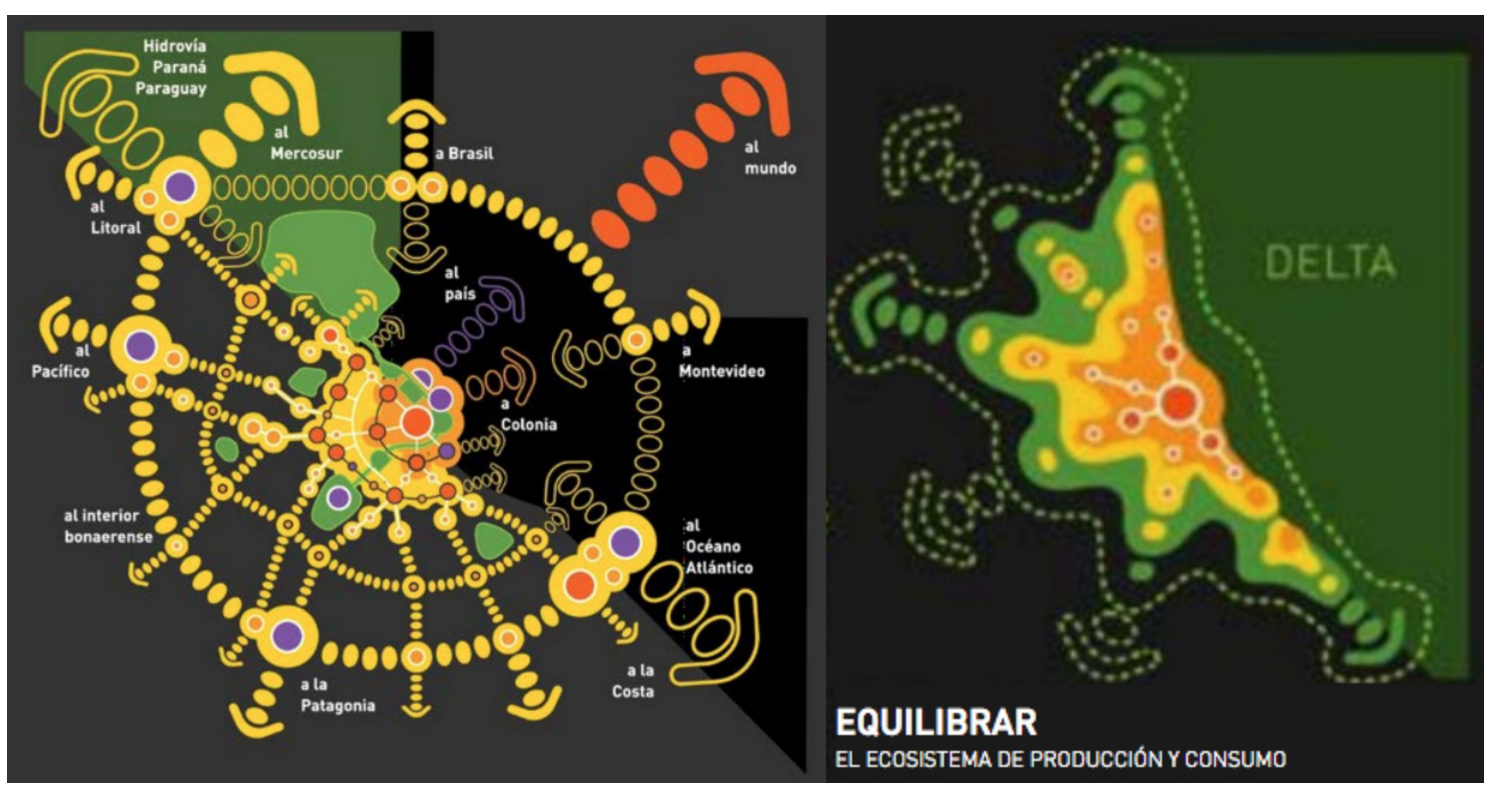

Fuente: Menini (2013)

Es decir que existe una dimensión económica considerable de los ríos (actividades portuarias, logísticas, turísticas...), inherente al funcionamiento de las metrópolis. El desafío es entonces combinar distintas lógicas (productividad agrícola, rentabilidad turística, tranquilidad residencial...), en una relación de combinación y no de competencia por los recursos (espacio, mano de obra, agua, mercado inmobiliario...) (Vanier, 2013). Ahora bien, la planificación no dispone todavía de conceptos lo suficientemente innovadores y robustos como para abordar una nueva generación de políticas públicas. En este marco, y como sostiene Vanier, el tercer espacio no conlleva problemas de división o recorte administrativo pero lleva a imaginar otras relaciones entre los poderes políticos territorializados y los espacios de vida en las dinámicas metropolitanas.

En cuanto a la dimensión medioambiental, a una escala amplia, el Delta del Paraná es de una importancia primordial para el mantenimiento de los equilibrios ecológicos, hidrológicos y geoquímicos del Río de la Plata y del AMBA. Además, la situación aguas abajo de Buenos Aires cuestiona el papel del Paraná para los equilibrios medioambientales de la metrópolis. En efecto, las industrias pesadas, las activida- des portuarias y agropecuarias se encuentran agua arriba y la calidad del agua que abastece el AMBA es (muy) poco controlada. A su vez, el Delta alberga poblaciones socialmente muy vulnerables con niveles de Necesidades Básicas Insatisfechas extremos (Massin, 2017). Por lo tanto, los desafíos de una transición hacia un desarrollo equilibrado del delta y de la metrópolis son muy grandes.

Para tal transición, el Paraná tiene que ser un elemento central para la planificación metropolitana. El delta inferior es, sin duda, el "verdadero pulmón verde" de la región (Subsecretaría de Urbanismo y Vivienda de la Provincia de Buenos Aires, 2007) y las modificaciones inducidas por el cambio climático refuerzan los desafíos de su gestión (Fanchette, 2006; Meyer \&Nijhuis, 2014). En las regiones metropolitanas, las zonas naturales, en particular los deltas, están afectadas directamente por los cambios planetarios, pero están también en posición de aportar soluciones innovadoras, al constituir espacios abiertos indispensables para la sostenibilidad de las metrópolis.

Respecto de la dimensión de representación visual y mental de la metrópolis, el río Para- 


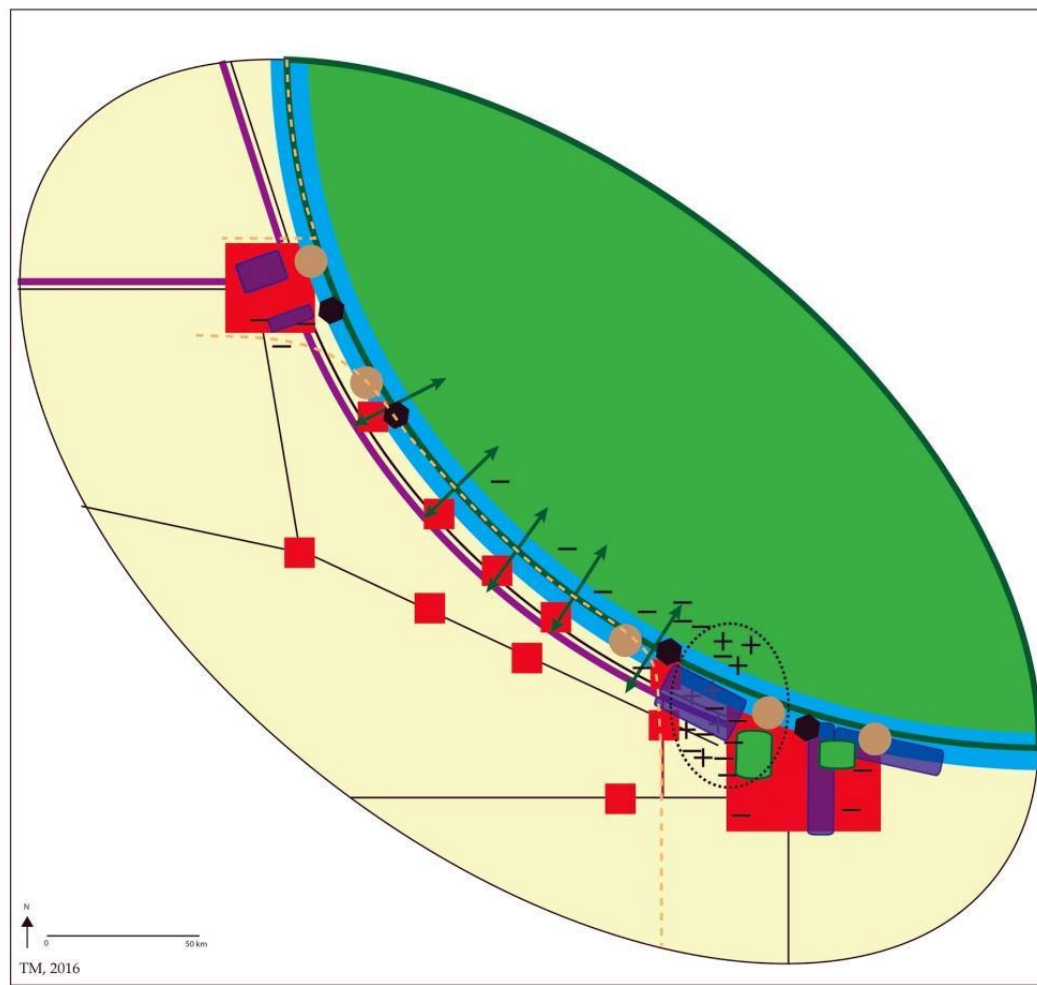

La metrópolis fluvial

Principales vulnerabilidades

- $\quad$ población muy vulnerable

zona de riesgo de inundación

espacio codiciado: urbanizaciones cerradas construidas sobre humedales

efluentes industriales principales ejes viales: movilidad individual y poco

Pistas de planificación

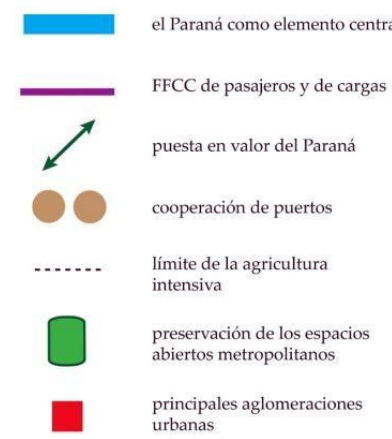

Fuente: elaboración propia

ná puede considerarse un elemento central prospectivo. En efecto, la complejidad metropolitana pensada desde los medios naturales permite la movilización de un imaginario colectivo. De este modo, el río Paraná se inscribe como soporte lineal de la metrópolis y su identidad geográfica coincide con la pertinencia industrial y portuaria. Como ejemplo, podemos citar a el caso del Grand Paris -término impreciso y adueñado por muchos actores y que agrupa varios componentes- que refleja la voluntad de promover la escala metropolitana. Los trabajos del Atelier International du Grand Paris permitieron hacer emerger la idea de considerar la metrópolis desde sus márgenes y la inversión de la mirada, para darle una identidad compartida por la mayoría de los actores. Especialmente, la propuesta de Grumbach que considera el Gran París hasta sus puertos de Ruan y Le Havre, a lo largo del Sena es muy eficiente a la hora de ampliar la mirada sobre la metrópolis parisina. Una de las críticas al proyecto (contra la voluntad de su autor) fue que se inscribe en un marco neo liberal global, con el cual las políticas de escalas inferiores se articulan. Así los principales argumentos políticos usados para defender el proyecto de Eje del Sena son la competencia económica (atractivo del territorio, integración portuaria, desarrollo de las actividades productivas) mientras que los argumentos sociales y medioambientales se encuentran relegados (Brennetot, Bussi\&Guermond, 2013). Son estas las dimensiones que destacamos en el modelo de metrópolis fluvial.

\section{La metrópolis fluvial o la representación me- tropolitana desde el río}

Se imponen dos pistas para integrar el Paraná en las reflexiones metropolitanas: crear un sentimiento de pertenencia colectiva y una identidad territorial. De esta forma, el Paraná debe tornarse el marco de una gran metrópolis donde lo urbano y lo natural estén reconciliados, donde no se vea más como un espacio codiciado por actores privados, sino como 
elemento unificador para todos los actores. El Paraná es el pretexto para pensar un equilibrio entre el urbanismo intenso y difuso, para pensar la forma de la metrópolis contemporánea en un esquema de metrópolis fluvial, que se inspira en el desarrollo metropolitano lineal proyectado en las décadas 1950 y 1960 pero de manera integrada. Somos conscientes que todo proyecto de planificación necesita una voluntad y un impulso políticos, tanto más fuerte cuanto más complejo es el proyecto, pero nos inscribimos en un enfoque prospectivo optimista y de largo plazo.

El modelo de metrópolis fluvial (fig. 9) retoma en parte las propuestas del Esquema Director año 2000 (Presidencia de la Nación, 1969) con la linealidad metropolitana sostenida por el transporte público y la integración del Delta, así como los Lineamientos Estratégicos para la Región Metropolitana de Buenos Aires (Subsecretaría de Urbanismo y Vivienda de la Provincia de Buenos Aires, 2007). Además, el modelo propone integrar el corredor a esta visión y le agrega nuevas dimensiones. Por un lado, el esquema tiene en cuenta las principales vulnerabilidades (poblaciones de pocos recursos y hogares con NBI, principales zonas inundables, espacios ecológicamente frágiles y codiciados por el desarrollo de urbanizaciones cerradas y principales zonas de desecho de efluentes industriales). Por otro lado, frente a ellas, presenta seis pistas de planificación:

- Considerar el Paraná como elemento central de la planificación metropolitana,
- Lo que implica también su consideración en los documentos locales de planificación,

- Hacer colaborar y cooperar los puertos: las futuras decisiones políticas en cuanto a la ubicación de las actividades portuarias metropolitanas van a ser capitales para el futuro de la metrópolis, creando un nuevo escenario metropolitano;

- Establecer reglas claras para evitar una agricultura intensiva en el Delta;

- Preservar los espacios abiertos metropolitanos, lo que necesita hacer evolucionar el sistema actual de área protegida, poco legible;

- Desarrollar un transporte público masivo, de calidad y accesible a todas las clases sociales, que estructure y "consolide" el corredor. Reafirmamos la importancia del transporte público en la estructuración territorial, si es pensado con un objetivo de eficiencia y de equidad social.

La figura de metrópolis fluvial permite ir en contra de la lógica jerárquica y centralizadora que se ha impuesto desde hace medio siglo; en cambio, presenta un sistema reticular y policéntrico en el cual las relaciones interurbanas juegan un papel decisivo. De este modo, la metropolización se ve como un conjunto de territorios circundante al foco metropolitano que ofrecen amenidades y ventajas para la población y las actividades. Así, estos espacios le ofrecen recursos a la aglomeración mientras se benefician de los servicios y equipamientos de la ciudad central, en una lógica de complementariedad y de articulación entre varios tipos de territorios.

\section{Bibliografía}

Albornoz Videla, G. (1950). Evita. Buenos Aires: Luis Lasserre.

Brennetot, A., Bussi, M. \&Guermond, Y. (2013). Le Grand Paris et l'axeSeine. Métropoles, 13.

Consejo del Plan Urbano Ambiental. (2000). Plan Urbano Ambiental, Documento final.

Dadon, J. (2003). Argentina, de espaldas al mar. Le Monde diplomatique, Edición Cono Sur, 53.

Recuperado de: http://www.ecologiamarina.com.ar/down/diplo.pdf

Della Paolera, C. (2010). Proyecto Epsilon. Revista de Arquitectura SCA, 238. 
Fanchette, S. (2006). De l'importance des liensgéographiephysique/géographiehumainepourcomprendre les risques de submersion des deltas surpeuplés. Hérodote, 2 (121), 6-18.

Recuperado de: https://www.cairn.info/revue-herodote-2006-2-page-6.htm

Forget, M. (2011). Le Paranaargentin, un fleuve du nouveau monde (Tesis doctoral).

Recuperado de: http://theses.univ-lyon2.fr/documents/lyon2/2011/forget_m

Forget, M. \&Bravard, J.P. (2011). The role of historicalsources in thefunctionalrepresentation of a river in the New World. The case of theargentinian Paraná. WaterHistory, 3 (3), 227-246.

INDEC. (1970). Censo Nacional de Población, Familias y Viviendas.

Latrubesse, E. (2008). Patterns of anabranchingchannels: theultimateend-memberadjustment of mega rivers. Geomorphology, 101, 130-145.

Recuperado de: https://www.unl.edu.ar/rcem2009/img/cursos/a1.pdf

Lefebvre, H. (1974). La production de l'espace. París: Anthropos.

Massin, T. (2017). El corredor metropolitano entre Buenos Aires y Rosario: un espacio complejo de la metropolización (Tesis doctoral).

Recuperado de: http://www.theses.fr/2017USPCA005/document

Ministerio de Infraestructura Vivienda y Servicios públicos de la Provincia de Buenos

Aires. (2006). Contribución al PET.

Menini, M. (2013). Las dinámicas del crecimiento urbano. Revista de Arquitectura SCA, 244.

Meyer, H. y Nijhuis, S. (2014). Urbanized deltas in transition. Amsterdam: TechnePress.

Moine, A. (2006). Le territoirecomme un systèmecomplexe: un concept opératoirepourl'aménagement et la géographie. L'EspaceGéographique, 2 (35), 115-132.

Recuperado de:

https://www.cairn.info/revue-espace-geographique-2006-2-page-115.htm

Monnet, J. (2001). Mexique, Amérique latine et Amériquesface à la métropolisation et à la mondialisation. Historiens et Géographes, 374, 369-378.

Municipalidad de Buenos Aires. (1962). Plan Regulador de la Ciudad de Buenos Aires.

Presidencia de la Nación. (1969). Esquema Director para el Año 2000. ORM.

Provincia de Buenos Aires. (2010). Estudio para la Formulación del Esquema Director Intermunicipal del Borde Metropolitano.

Subsecretaría de Ambiente y Desarrollo Sustentable de la Nación. (2002). Proyecto Epsilon.

Subsecretaría de Urbanismo y Vivienda de la Provincia de Buenos Aires. (2007). Lineamientos Estratégicos para la Región Metropolitana de Buenos Aires.

Recuperado de:

https://www.mininterior.gov.ar/planificacion/pdf/planes-reg/Lineamientos-Estrategicos-para-la-Region-Metropolitana-de-Buenos-Aires.pdf

Vanier, M. (2003). Le périurbain à l'heure du crapaudbuffle :tiersespace de la nature, nature du tiersespace. Revue de GéographieAlpine, 91 (4), 79-89.

Recuperado de: https://www.persee.fr/doc/rga_0035-1121_2003_num_91_4_2264

Vanier, M. (2000). Qu'est-ce que le tiersespace ?Territorialitéscomplexes et constructionpolitique. Revue de GéographieAlpine, 88 (1), 105-113.

Recuperado de: https://www.persee.fr/doc/rga_0035-1121_2000_num_88_1_4626

Walsh, R. (1995). El violento oficio de escribir: obra periodística (1953-1977). Buenos Aires: Planeta. 
